\title{
Efficacy of Enteral Supplementation Enriched with Glutamine, Fiber, and Oligosaccharide on Mucosal Injury following Hematopoietic Stem Cell Transplantation
}

\author{
Satoshi Iyama ${ }^{a, b}$ Tsutomu Sato $^{a}$ Hiroomi Tatsumi $^{\mathrm{b}, c}$ \\ Akari Hashimoto $^{a}$ Ayumi Tatekoshi $^{\mathrm{a}}$ Yusuke Kamihara $^{\mathrm{a}}$ \\ Hiroto Horiguchi ${ }^{a}$ Soushi Ibata ${ }^{a} K$ Kaoru Ono ${ }^{a}$ Kazuyuki Murase ${ }^{a}$ \\ Kohichi Takada $^{a}$ Yasushi Sato ${ }^{a}$ Tsuyoshi Hayashi $^{a}$ Koji Miyanishi ${ }^{a}$ \\ Emi Akizuki ${ }^{\text {b, }}$ Takayuki Nobuoka ${ }^{\text {b, }}$ Toru Mizugichid ${ }^{d}$ Rishu Takimoto ${ }^{a}$ \\ Masayoshi Kobune $^{a} K_{\text {Koichi Hirata }}{ }^{b} d \quad$ Junji Kato ${ }^{a}$ \\ ${ }^{a}$ Department of Medical Oncology and Hematology, ${ }^{b}$ Nutritional Support Team, \\ ${ }^{\mathrm{C}}$ Department of Traumatology and Critical Care Medicine, Intensive Care Unit, and \\ ${ }^{d}$ Department of Surgery, Surgical Oncology and Science, Sapporo Medical University \\ School of Medicine, Sapporo, Japan
}

\section{Key Words}

Glutamine · Fiber · Oligosaccharide · GFO $\cdot$ Mucosal injury $\cdot$ Hematopoietic stem cell transplantation

\begin{abstract}
The combination of glutamine, fiber and oligosaccharides (GFO) is thought to be beneficial for alleviating gastrointestinal mucosal damage caused by chemotherapy. A commercial enteral supplementation product (GFO) enriched with these 3 components is available in Japan. We performed a retrospective study to test whether oral GFO decreased the severity of mucosal injury following hematopoietic stem cell transplantation (HSCT). Of 44 HSCT patients, 22 received GFO and 22 did not. Severity of diarrhea/mucositis, overall survival, weight loss, febrile illness/documented infection, intravenous hyperalimentation days/ hospital days, engraftment, acute and chronic GVHD, and cumulative incidence of relapse were studied. Sex, age, performance status, diagnosis, disease status, and treatment variables were similar in both groups. There were fewer days of diarrhea grade 3-4 in patients receiving GFO than in those who did not (0.86 vs. 3.27 days); the same was true for days of
\end{abstract}


mucositis grade $3-4$ (3.86 vs. 6.00 days). Survival at day 100 was $100 \%$ in the GFO group, but only $77.3 \%$ for the patients not receiving GFO ( $p=0.0091$, log-rank test). Weight loss and the number of days of intravenous hyperalimentation were better in the GFO group $(p<0.001$ and $p=0.0014$, respectively). Although not significant, less gut bacterial translocation with Enterococcus species developed in the GFO group ( $p=0.0728)$ than in the non-GFO group. Other outcomes were not affected. To the best of our knowledge, this is the first comparative clinical study of GFO supplementation to alleviate mucosal injury after allo-HSCT. We conclude that glutamine, fiber and oligosaccharide supplementation is an effective supportive therapy to decrease the severity of mucosal damage in HSCT. @ $2014 \mathrm{~S}$. Karger AG, Basel

\section{Introduction}

Gastrointestinal mucosal injury is one of the most serious complications resulting from the conditioning regimens required for allogeneic hematopoietic stem cell transplantation (HSCT). It causes significant morbidity and may affect prognosis, because disruption of the gastrointestinal mucosal barrier facilitates translocation of microorganisms and/or endotoxins into the blood stream and increases mortality [1]. In this context, appropriate mitigation of gastrointestinal mucositis might improve the survival rate after HSCT.

Earlier studies comparing nutritional support with and without glutamine supplementation for patients with mucositis after HSCT were conducted because many investigators considered glutamine to be essential in critical illnesses such as radiation-induced colitis, sepsis, trauma, and burns [2-4]. These and other studies suggest that glutamine may exert a protective effect on the gut mucosal barrier and increase short-term survival after HSCT [57]. Standard total parenteral nutrition (TPN) does not contain glutamine because of its instability during heat sterilization and prolonged storage [8]. Additionally, glutamine for TPN, such as L-alanyl-L-glutamine dipeptide, is not available in Japan.

Both dietary fiber and oligosaccharide are also promising candidates for dietary supplements protecting against gastrointestinal mucosal injury induced by HSCT conditioning regimens. Dietary fiber plays an important role in controlling diarrhea, improving the restoration of bowel function, and reducing infection, thereby improving the prognosis of critically ill patients [9]. Oligosaccharide is thought to have beneficial effects by suppressing pathogenic bacteria in the colon [10]. Thus, the combination of glutamine, fiber and oligosaccharides (GFO) could be expected to protect against gastrointestinal mucosal injury in HSCT patients. Dietary fiber can also be fermented into short-chain fatty acids by bowel microflorae, which can protect the intestinal barrier and prevent bacterial translocation [9].

Currently, a commercial enteral GFO supplementation product is available in Japan. Recent investigations have revealed that this GFO preparation prevented gut bacterial translocation to the mesenteric lymph nodes in a murine model of bacterial overgrowth [11], and Joo et al. [9] reported that GFO has suppressive effects on mucosal damage in a murine ulcerative colitis model [12].

This study tests the hypothesis that enteral supplementation with GFO ameliorates gut injury induced by HSCT conditioning regimens and improves patient short-term survival in a retrospective analysis. 


\section{Case Reports in Oncology}

\begin{tabular}{l|l}
\hline Case Rep Oncol 2014;7:692-699 \\
\hline DOI: 10.1159/000368714 & $\begin{array}{l}\text { ○ 2014 S. Karger AG, Basel } \\
\text { www.karger.com/cro }\end{array}$ \\
\hline
\end{tabular}

Iyama et al.: Efficacy of Enteral Supplementation Enriched with Glutamine, Fiber, and Oligosaccharide on Mucosal Injury following Hematopoietic Stem Cell Transplantation

\section{Patients and Methods}

\section{Patients}

Patients who underwent stem cell transplantation at Sapporo Medical University Hospital between January 2009 and April 2011 were analyzed retrospectively with institutional review board approval. Patients were eligible for transplantation if they had any hematological malignancies at high risk for relapse and if suitable related or unrelated bone marrow/peripheral blood donors had been available within a reasonable period relative to their disease condition. Patients who had end-stage cardiac dysfunction (left ventricular ejection fraction $<35 \%$ ), pulmonary dysfunction (Sp02 $<90 \%$ in room air), or active serious infection at the time of transplantation were not eligible. All patients gave written informed consent.

\section{Conditioning Regimens and Graft-versus-Host Disease Prophylaxis}

Conditioning regimens were either conventional or reduced-intensity conditioning (RIC) regimens. Conventional conditioning included the combination of busulfan $16 \mathrm{mg} / \mathrm{kg}$ and cyclophosphamide (CY) $120 \mathrm{mg} / \mathrm{kg}$ or total body irradiation $12 \mathrm{~Gy}$ and CY $120 \mathrm{mg} / \mathrm{kg}$. RIC involved the use of fludarabine as a substitute for or in association with the conventional regimen drugs. Graft-versus-host disease (GVHD) prophylaxis regimens included the combination of FK506 and short-term methotrexate (MTX) or cyclosporine and short-term MTX. The first-line treatment for acute GVHD was prednisolone 1-2 mg/kg for all patients.

\section{GFO administration}

GFO was purchased from Otsuka Pharmaceutical Co., Ltd. (Tokushima, Japan). One pack of GFO (15 g) has $36 \mathrm{kcal}$ and contains $3 \mathrm{~g}$ of glutamine, $5 \mathrm{~g}$ of dietary fiber, $1.5 \mathrm{~g}$ of oligosaccharide, and $1.2 \mathrm{mg}$ of sodium. Two packages of GFO dissolved in $200 \mathrm{ml}$ of water were administered to patients orally 3 times per day beginning 7 days prior to the start of conditioning and continued until 28 days after transplantation. Administration was stopped if vomiting occurred. TPN, conditioning chemotherapy, medication and transfusions were administered through a central venous catheter. While the neutrophil count remained $<0.5 \times 10^{9} /$ l, patients were nursed in reversed isolation.

\section{Evaluation of Enteral Supplementation Enriched with GFO}

Diarrhea and oral mucositis were assessed using Common Terminology Criteria for Adverese Events (CTCAE) version 4.0. Diarrhea was classified as grade I when the incidence was below 4 episodes per day relative to baseline, as grade II when the incidence was 4-6 episodes per day over baseline, as grade III when the incidence was $>7$ episodes per day over baseline, as grade IV when life-threatening consequences occurred, and as grade $V$ when the patient died. Mucositis (oral) was classified as grade I when the patients was asymptomatic or only had mild symptoms (intervention not indicated), as grade II when moderate pain was experienced, not interfering with oral intake (modified diet indicated), as grade III when severe pain interfering with oral intake occurred, as grade IV when life-threatening consequences were found (urgent intervention indicated), and as grade $\mathrm{V}$ when the patient died. The mean and highest grades and durations were then calculated for analysis. The number of days and severity of diarrhea, mucositis, weight loss, fever $\left(>38.5^{\circ} \mathrm{C}\right)$, TPN, episodes of microbiologically-documented infections, and hospital days were collected.

\section{Selection of Matched Controls and Matching Variables}

A matched-pair control group (no GFO supplement) for patients who used GFO was obtained from our historical database from 2006 to 2008. The controls were individually matched to the cases at a 1:1 ratio. Matching was attempted for the following criteria applied in the order they are listed: age at transplantation ( $<55$ vs. $\geq 55$ years), ECOG performance status ( 0 or 1 ), disease status (standard vs. high risk; i.e. patients with acute leukemia, chronic myeloid leukemia, malignant lymphoma in complete remission, and myelodysplastic syndromes (refractory anemia) were categorized as standard risk, and all others as high risk), pretransplant conditioning (conventional vs. RIC), GVHD prophylaxis (FK- vs. cyclosporine-based), and graft source (bone marrow vs. peripheral blood). To avoid any potential selection bias, matching was blinded, and only the patients' ID and pretreatment variables were known. 
Statistical Analysis

Data were entered into a computerized database and analyzed by either Fisher's exact test or a $\chi^{2}$ test for categorical data and the Mann-Whitney $U$ test for non-categorical data. The data analyzed between groups were as follows: (1) average diarrhea/mucositis score; (2) overall survival; (3) body weight loss; (4) febrile illness/documented infection; (5) intravenous hyperalimentation days/hospital days; (6) time to neutrophil engraftment; (7) incidences of acute and chronic GVHD, and (8) cumulative incidence of relapse.

The probabilities of overall survival were estimated and plotted using the Kaplan-Meier method. Data analysis was performed using GraphPad Prism version 5.0b (GraphPad Software Inc., San Diego, Calif., USA). The level of significance in all cases was set at $\mathrm{p}<0.05$.

\section{Results}

\section{Patient Characteristics}

Twenty-two patients who underwent stem cell transplantation and received GFO supplementation were analyzed. A matched-pair control group (no GFO supplementation) was obtained from our historical database. The characteristics of these two groups are summarized in table 1 . There was no difference between them with regard to sex $(p=0.75)$, age $(p=$ $0.53)$, ECOG performance status $(p=0.62)$, underlying diagnoses $(p=0.55)$, disease status $(p=1.0)$, conditioning regimen $(p=0.93)$, GVHD prophylaxis $(p=0.66)$, and graft source $(\mathrm{p}=1.0)$.

\section{Clinical Outcomes}

Clinical outcomes are shown in table 2. There were no differences in the maximum diarrhea $(p=0.68)$ or mucositis $(p=0.20)$ grades. However, a statistically significant reduction in the number of days of diarrhea grade $3-4(p=0.001)$ or grade $2(p=0.0001)$ and days of mucositis grade $3-4(p=0.033)$ was apparent in the GFO-supplemented group. GFO administration also prevented weight loss $(\mathrm{p}<0.001)$ and reduced the number of days of intravenous hyperalimentation $(p=0.001)$. There were no differences in the number of days with fever $\left(>38.5^{\circ} \mathrm{C} ; \mathrm{p}=0.41\right)$ or microbiologically-documented infections $(\mathrm{p}=0.71)$, all of which were bloodstream infections: 3 methicillin-resistant Staphylococcus aureus (MRSA) and 1 methicillin-resistant Staphylococcus epidermidis (MRSE) in the GFO group, and 2 Enterococcus faecium, 1 Enterococcus faecalis, 1 methicillin-resistant $S$. aureus, and 1 methicillin-resistant $S$. epidermidis in the group without GFO supplementation. GFO administration did not affect the number of hospital days $(\mathrm{p}=0.88)$. Next, we analyzed the impact of GFO on clinical parameters of HSCT, especially the time to engraftment, incidence and severity of acute/chronicGVHD and relapse rate. We found no differences between the two groups in this respect (data not shown). However, an apparent benefit was observed in the GFO group regarding the survival rate 100 days after HSCT (100 vs. $77.3 \%, p=0.0091$, log-rank test) as shown in figure 1 . There were 5 deaths during the first 100 days after HSCT in the group without GFO due to refractory disease in 2 cases, sepsis in 2 cases, and pneumonia in 1 case. Median survival time was 530 versus 416 days $(p=0.6871)$ in the GFO and non-GFO group, respectively.

\section{Discussion}

In the present study, we documented the beneficial effects of GFO in protecting against mucosal injury, decreasing weight loss and days of intravenous hyperalimentation, and 
increasing short-term survival after HSCT (table 2; fig. 1). Conditioning chemotherapy and irradiation for HSCT evoke severe diarrhea and this adversely affected early therapy-related mortality. We observed that severe diarrhea was particularly improved in the GFOsupplemented group. Thus, it was considered that a reduction of therapy-related early mortality had been achieved. In fact, there were 2 patients who died from Entercoccus species infection due to bacterial translocation in the early phase after HSCT in the non-GFO group, although GFO supplementation neither suppressed the incidence of documented infection nor shortened the febrile period (table 2). However, gut bacterial translocation, such as that of Enterococcus species (E. faecium or E. faecalis), tended not to develop in the GFO group ( $p=0.0728$ relative to the control group). This suggested that GFO supplementation reduces gut mucosal injury and sepsis caused by enterococci in HSCT patients. Oligosaccharides included in GFO are prebiotics which have beneficial effects on commensal bacteria. Furthermore, we used a lactobacillus preparation (Biofermin-R, Biofermin Pharmaceutical Co. Ltd., Japan) as a probiotic in all patients. Since we observed mucosal injury less frequent and of shorter duration, we conclude that GFO and probiotics have synergistic effects (i.e. they are so-called 'synbiotics') and were able to ameliorate mucosal injury caused by the conditioning regimen.

Contrasting observations regarding the efficacy of oral glutamine [13] for amelioration of chemotherapy-associated mucositis could be related to a different route and schedule of glutamine administration in those studies. Anderson et al. [1] reported the efficacy of lowdose oral glutamine on oral mucosal injury during autologous bone marrow transplantation [14]. The dose of glutamine used in our study is comparatively higher than the average dietary intake (2-5 g/day) and almost the same as used by Anderson et al. A 2-gram oral dose of glutamine can significantly raise $(0.1 \mathrm{mM}$ difference) blood glutamine levels for about $1 \mathrm{~h}$ [15]. Since GFO contains $3 \mathrm{~g}$ of glutamine per pack, effects seen with the dose used in our study may be related to absorbed glutamine. Patients on GFO received an average of $32.4 \mathrm{~g}$ (2.16 per pack) per day, containing $6.48 \mathrm{~g}$ of glutamine, $10.8 \mathrm{~g}$ of dietary fiber, and $3.24 \mathrm{~g}$ of oligosaccharide. In the present study, using a relatively high dose of glutamine, fiber, and oligosaccharide, no GFO-related toxicity was found. GFO supplementation was well tolerated by the patients, and no allergic reactions were documented.

Oral glutamine appears to reduce GVHD and i.v. glutamine may increase the risk of relapse, according to a meta-analysis of studies of glutamine supplementation [7]. In our study, GFO supplementation neither suppressed the incidence of acute or chronic GVHD nor increased the relapse rate. However, these findings are based on a small number of patients. For definitive conclusions, larger well-designed prospective trials are required.

To the best of our knowledge, this is the first retrospective comparative clinical study of mucosal injury in allogeneic stem cell transplantation using GFO. We conclude that GFO supplementation is an effective supportive therapy to decrease the severity of mucosal injury in HSCT, which is a cause of morbidity associated with this treatment. Comparison of GFO with glutamine alone is planned for the future.

\section{Acknowledgements}

We appreciate the assistance of the physicians, physician assistants, nurses and support staff on the transplantation units and nutritional support team. 


\section{Case Reports in Oncology}

\begin{tabular}{l|l}
\hline DOI: $10.1159 / 000368714$ & $\begin{array}{l}\text { C 2014 S. Karger AG, Basel } \\
\text { www.karger.com/cro }\end{array}$ \\
\hline
\end{tabular}

Iyama et al.: Efficacy of Enteral Supplementation Enriched with Glutamine, Fiber, and Oligosaccharide on Mucosal Injury following Hematopoietic Stem Cell Transplantation

\section{Disclosure Statement}

This paper has not been published or submitted for publication elsewhere. All authors have contributed significantly and are in agreement with the content of the manuscript. We declare that there are no financial or other relationships that may represent a conflict of interest.

\section{References}

1 Anderson PM, Ramsay NK, Shu XO, Rydholm N, Rogosheske J, Nicklow R, Weisdorf DJ, Skubitz KM: Effect of low-dose oral glutamine on painful stomatitis during bone marrow transplantation. Bone Marrow Transplant 1998;22:339-344.

-2 Aquino VM, Harvey AR, Garvin JH, Godder KT, Nieder ML, Adams RH, Jackson GB, Sandler ES: A double-blind randomized placebo-controlled study of oral glutamine in the prevention of mucositis in children undergoing hematopoietic stem cell transplantation: a pediatric blood and marrow transplant consortium study. Bone Marrow Transplant 2005;36:611-616.

-3 Azuma H, Mishima S, Oda J, Homma H, Sasaki H, Hisamura M, Ohta S, Yukioka T: Enteral supplementation enriched with glutamine, fiber, and oligosaccharide prevents gut translocation in a bacterial overgrowth model. Journal Trauma 2009;66:110-114.

4 Crowther M, Avenell A, Culligan DJ: Systematic review and meta-analyses of studies of glutamine supplementation in haematopoietic stem cell transplantation. Bone Marrow Transplant 2009;44:413-425.

5 da Gama Torres HO, Vilela EG, da Cunha AS, Goulart EM, Souza MH, Aguirre AC, Azevedo WM, Lodi FM, Silva AA, Bittencourt HN: Efficacy of glutamine-supplemented parenteral nutrition on short-term survival following allo-SCT: a randomized study. Bone Marrow Transplant 2008;41:1021-1027.

-6 Gibson GR, Roberfroid MB: Dietary modulation of the human colonic microbiota: introducing the concept of prebiotics. Journal Nutr 1995;125:1401-1412.

7 Jebb SA, Osborne RJ, Maughan TS, Mohideen N, Mack P, Mort D, Shelley MD, Elia M: 5-Fluorouracil and folinic acid-induced mucositis: no effect of oral glutamine supplementation. BriJ Cancer 1994;70:732-735.

8 Johansson JE, Ekman T: Gastro-intestinal toxicity related to bone marrow transplantation: disruption of the intestinal barrier precedes clinical findings. Bone Marrow Transplant 1997;19:921-925.

-9 Joo E, Yamane S, Hamasaki A, Harada N, Matsunaga T, Muraoka A, Suzuki K, Nasteska D, Fukushima T, Hayashi T, Tsuji H, Shide K, Tsuda K, Inagaki N: Enteral supplement enriched with glutamine, fiber, and oligosaccharide attenuates experimental colitis in mice. Nutrition 2013;29:549-555.

-10 Kucuktulu E, Guner A, Kahraman I, Topbas M, Kucuktulu U: The protective effects of glutamine on radiationinduced diarrhea. Support Care Cancer 2013;21:1071-1075.

11 Lacey JM, Wilmore DW: Is glutamine a conditionally essential amino acid? Nutr Rev 1990;48:297-309.

12 Oudemans-van Straaten HM, Bosman RJ, Treskes M, van der Spoel HJ, Zandstra DF: Plasma glutamine depletion and patient outcome in acute ICU admissions. Intensive Care Med 2001;27:84-90.

$\checkmark 13$ Skubitz KM, Anderson PM: Oral glutamine to prevent chemotherapy induced stomatitis: a pilot study. J Lab Clin Med 1996;127:223-228.

14 Welbourne TC: Increased plasma bicarbonate and growth hormone after an oral glutamine load. Am J Clin Nutr 1995;61:1058-1061.

15 Yang G, Wu XT, Zhou Y, Wang YL: Application of dietary fiber in clinical enteral nutrition: a meta-analysis of randomized controlled trials. World J Gastroenterol 2005;11:3935-3938. 
Table 1. Characteristics of transplant recipients

\begin{tabular}{|c|c|c|c|}
\hline \multirow[t]{2}{*}{ Characteristics } & \multicolumn{2}{|l|}{ Patients } & \multirow[t]{2}{*}{$\mathrm{p}$} \\
\hline & GFO & Controls & \\
\hline Sex & & & 0.7470 \\
\hline Male & 16 & 14 & \\
\hline Female & 6 & 8 & \\
\hline \multicolumn{3}{|l|}{ Age, years } & 0.5256 \\
\hline Median (range) & \multicolumn{2}{|c|}{$52.5(21-47(19-62)$} & \\
\hline \multicolumn{3}{|l|}{$\begin{array}{l}\text { Age distribution, } \\
\text { years }\end{array}$} & 1.0 \\
\hline$<55$ & $5(23)$ & $5(23)$ & \\
\hline$\geq 55$ & $17(77)$ & $17(77)$ & \\
\hline \multicolumn{3}{|c|}{ ECOG performance status } & 0.6163 \\
\hline 0 & $21(95)$ & $20(91)$ & \\
\hline 1 & $1(5)$ & $2(9)$ & \\
\hline \multicolumn{3}{|l|}{ Diagnosis } & 0.5540 \\
\hline AML & $9(41)$ & $9(41)$ & \\
\hline MDS & $5(23)$ & $4(18)$ & \\
\hline ALL & $4(18)$ & $3(14)$ & \\
\hline CML & $1(5)$ & $0(0)$ & \\
\hline CLL & $1(5)$ & $0(0)$ & \\
\hline ML & $1(5)$ & $5(23)$ & \\
\hline MPD & $1(5)$ & $1(5)$ & \\
\hline \multicolumn{3}{|l|}{ Disease status } & 1.0 \\
\hline Standard risk & $10(45)$ & $11(50)$ & \\
\hline High risk & $12(55)$ & $11(50)$ & \\
\hline
\end{tabular}

\section{Conditioning}

Conventional

\begin{tabular}{crr} 
CY+TBI & 5 & 4 \\
BU+CY & 2 & 2 \\
RIC (Flu based) & 15 & 16 \\
\hline
\end{tabular}

\begin{tabular}{lrrr}
\hline GVHD prophylaxis & & & 0.6640 \\
FK506 + MTX & 18 & 20 & \\
CSP + MTX & 4 & 2 & 1.0 \\
\hline Graft source & & & \\
Bone marrow & 21 & 21 & \\
Peripheral blood & 1 & 1 &
\end{tabular}

Values are presented as n (\%) or median (range). AML = Acute myeloid leukemia; MDS = myelodysplastic syndromes; ALL = acute lymphoblastic leukemia; $\mathrm{CML}=$ chronic myeloid leukemia; $\mathrm{CLL}=$ chronic lymphocytic leukemia; $\mathrm{ML}=$ malignant lymphoma; $\mathrm{MPD}=$ myeloproliferative diseases; $\mathrm{TBI}=$ total body irradiation; $\mathrm{BU}=$ busulfan; Flu = fludarabine; $\mathrm{CSP}=$ cyclosporine. 
Table 2. Clinical outcomes

\begin{tabular}{lccc}
\hline & GFO & Non-GFO & $\mathrm{p}$ \\
\hline Diarrhea grade (max. grade score) & 2.00 & 2.68 & 0.0677 \\
$\quad$ Days of diarrhea grade $>3$ & 0.86 & 3.27 & 0.0009 \\
$\quad$ Days of diarrhea grade $>2$ & 3.73 & 7.68 & 0.0001 \\
Mucositis grade (max grade score) & 1.55 & 2.05 & 0.2000 \\
$\quad$ Days of mucositis grade $>3$ & 3.86 & 6.00 & 0.0330 \\
Body weight loss, $\mathrm{kg}$ & 2.15 & 6.42 & $<0.0001$ \\
Days of intravenous hyperalimentation & $36.91(0-125)$ & $54.14(21-82)$ & 0.0014 \\
Days with fever $\left(>38.5^{\circ} \mathrm{C}\right)$ & 0.73 & 1.41 & 0.4096 \\
Ratio of microbiologically documented infections & $4 / 22$ & $5 / 22$ & 0.7086 \\
Hospital days & $81.18(39-148)$ & $78.00(24-127)$ & 0.8787 \\
\hline
\end{tabular}

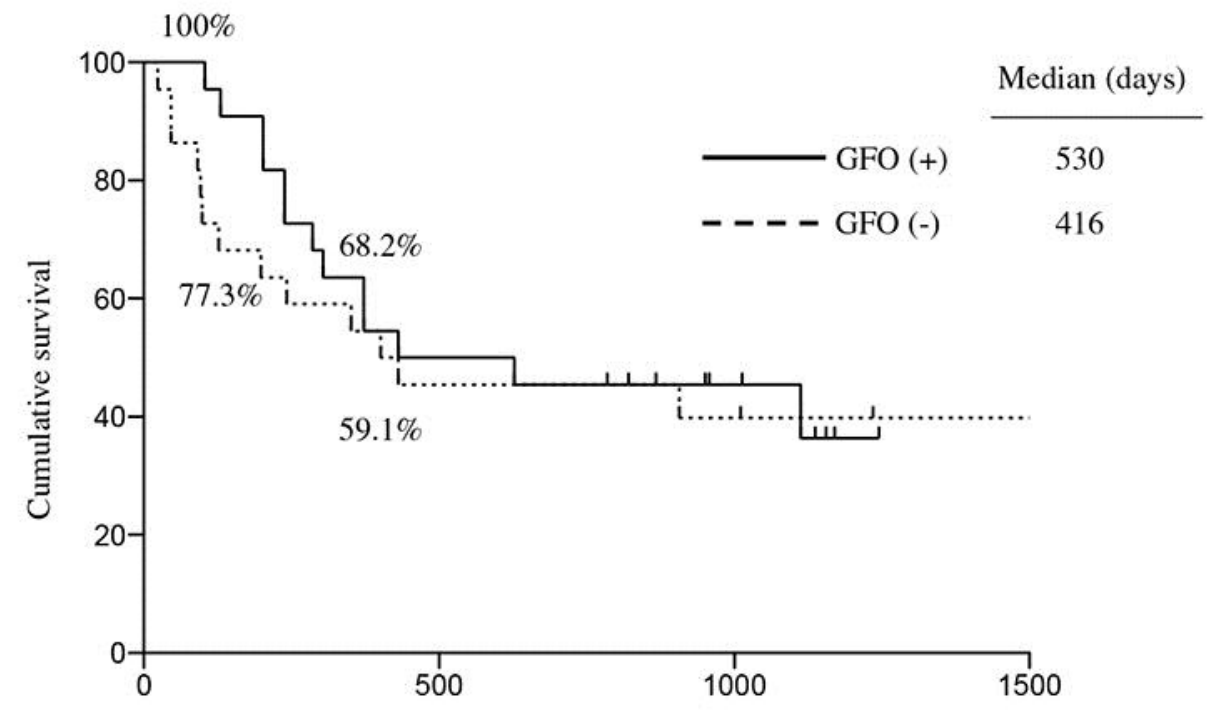

Time (days)

Fig. 1. Overall survival. 